\section{Antimicrobial Lock Solutions in the Prevention of Catheter-Related Bloodstream Infections in Patients Receiving Hemodialysis}

To the Editor-There is increasing evidence that antimicrobial locks applied within the catheter lumen are effective at preventing catheter-related bloodstream infection (CRBSI), a source of substantial morbidity and mortality in patients receiving hemodialysis (HD). ${ }^{1}$ Therefore, we read with interest the recently published review article by John Boyce that, in addition to providing a comprehensive presentation of measures and recommendations for the prevention of CRBSI, reviewed the trials of antimicrobial lock solutions as a strategy for reducing the incidence of such infections in patients receiving HD. ${ }^{2}$ Our own trial ${ }^{3}$ was reported in Table 3 of the publication and throughout the article as an observational study, which is apparently incorrect, and we would like to clarify this issue. Furthermore, data for our study group patients were presented in a rather confusing way in the table, and this also needs to be elucidated. Finally, please allow us to kindly make some remarks on the recommendations given by the author regarding the specific antimicrobial lock solutions that should be considered for use in patients receiving HD through a catheter.

In our study, ${ }^{3} 119$ patients with chronic illness who received dialysis via a temporary uncuffed or nontunneled HD catheter were randomly assigned to receive interdialytic catheter locking with either gentamicin-heparin $(40 \mathrm{mg} / \mathrm{mL}$ gentamicin and $5,000 \mathrm{U} / \mathrm{mL}$ unfractionated heparin; ratio, $1: 3$ ) or taurolidine-citrate $(1.35 \%$ taurolidine and $4 \%$ sodium citrate; TauroLock [TauroPharm]) at the end of each dialysis session and continuously after catheter insertion. Fifty-eight wellmatched patients who had received HD via an uncuffed catheter in a previous period were used as historical controls. The catheter lock solution in the historical control group contained unfractionated heparin alone at a concentration of $5,000 \mathrm{U} / \mathrm{mL}$. Therefore, this is a single-center, open-label, prospective, randomized study that was conducted to compare gentamicin-heparin with taurolidine-citrate as catheter lock solutions in terms of CRBSI prevention and catheter thrombosis in patients receiving chronic HD who require an uncuffed catheter as temporary vascular access until a permanent vascular access becomes available. Taking into consideration the high CRBSI rates observed in our heparinalone group and the fact that the preventive use of antimicrobial lock solutions is recommended by international guidelines, our decision was to exclude the heparin-alone control group from the randomization and to use a historical group instead.
In the conclusion of his review article, Boyce ${ }^{2}$ recommended the use of either gentamicin in $4 \%$ sodium citrate or a combination of sodium citrate-methylene blue-propylparaben as antimicrobial lock solutions, although no supporting data from the literature were provided. To the best of our knowledge, the currently available data do not enable a difference in efficacy to be identified between different solutions, because they have not been compared in a head-tohead, randomized controlled trial. Indeed, our study ${ }^{3}$ was the first randomized trial that directly compared gentamicinheparin, one of the most commonly used antibiotic-based lock solutions, and taurolidine-citrate, a broad-spectrum antimicrobial agent, as catheter locks in patients receiving HD. Although taurolidine-citrate appeared to be a safe and effective alternative to the established antimicrobial agents, our study failed to demonstrate its superiority in terms of CRBSI prevention. Furthermore, other recent reviews have appeared to advocate the use of lock solutions containing low concentrations of citrate in conjunction with taurolidine as the best choice at the moment, although the lack of sufficient efficacy data and clinical experience is recognized. ${ }^{4,5}$ Indeed, the use of such nonantibiotic locks would be a desirable solution to address the problem of antibiotic resistance.

We fully endorse the recommendation made by Boyce ${ }^{2}$ for the routine interdialytic use of an antimicrobial lock solution in patients who receive HD through a catheter, because this strategy will definitely contribute to the reduction of infectious complications, which remain a major clinical problem in this patient population and cause substantial cost burden to the healthcare system. However, additional comparative studies are needed to possibly clarify the ideal lock solution. In any case, we should bear in mind that the risk-benefit ratio of some antimicrobial lock solutions is not clear and that their long-term prognostic implications are uncertain.

\section{ACKNOWLEDGMENTS}

Potential conflicts of interest. All authors report no conflicts of interest relevant to this article. All authors submitted the ICMIE Form for Disclosure of Potential Conflicts of Interest, and the conflicts that the editors consider relevant to this article are disclosed here.

Vassilis Filiopoulos, MD; ${ }^{1}$ Dimitrios Hadjiyannakos, MD; ${ }^{1}$ Dimosthenis Vlassopoulos, $\mathrm{MD}^{1}$

Affiliation: 1. Nephrology Department, Amalia Fleming General Hospital, Athens, Greece.

Address correspondence to Vassilis Filiopoulos, MD, Department of Nephrology, Amalia Fleming General Hospital, 25th Martiou 14, 15127, Athens, Greece (vassilis.filiopoulos@hotmail.com).

Infect Control Hosp Epidemiol 2013;34(3):329-330

(C) 2013 by The Society for Healthcare Epidemiology of America. All rights reserved. 0899-823X/2013/3403-0019\$15.00. DOI: $10.1086 / 669516$ 


\section{REFERENCES}

1. Vanholder R, Canaud B, Fluck R, et al. Diagnosis, prevention and treatment of hemodialysis catheter-related bloodstream infections (CRBSI): a position statement of European Renal Best Practice (ERBP). NDT plus 2010;3:234-246.

2. Boyce JM. Prevention of central line-associated bloodstream infections in hemodialysis patients. Infect Control Hosp Epidemiol 2012;33:936-944.

3. Filiopoulos V, Hadjiyannakos D, Koutis I, et al. Approaches to prolong the use of uncuffed hemodialysis catheters: results of a randomized trial. Am J Nephrol 2011;33:260-268.

4. Betjes MG. Prevention of catheter-related bloodstream infection in patients on hemodialysis. Nat Rev Nephrol 2011;7:257-265.

5. Lok CE, Mokrzycki MH. Prevention and management of catheter-related infection in hemodialysis patients. Kidney Int 2011; 79:587-598.

\section{Reply to Filiopoulos et al}

To the Editor-I wish to thank Filiopoulos et $\mathrm{a}^{1}$ for their thoughtful comments regarding my recent article dealing with prevention of central line-associated bloodstream infection (CLABSI) in patients receiving hemodialysis. ${ }^{2}$ The authors point out that their study, cited in Table 3 of my review article, was summarized in a somewhat confusing manner. I referred to their study as an observational one. In fact, Filiopoulos et $\mathrm{al}^{1}$ randomized 60 patients to receive gentamicin-heparin and 59 patients to receive taurolidine-citrate. ${ }^{3}$ However, unlike a number of other recently conducted randomized controlled trials that involved contemporary controls, the Filiopoulos et $\mathrm{al}^{1}$ trial compared patients who received the 2 new study regimens with 58 historical control subjects.

The authors also state, "Boyce recommended the use of either gentamicin in $4 \%$ sodium citrate or a combination of sodium citrate-methylene blue-propylparaben as antimicrobial lock solutions, although no supporting data from the literature were provided." $1(\mathrm{pXXX})$ On the contrary, the randomized controlled trials by Moran et $\mathrm{al}^{4}$ and by Maki et $\mathrm{al}^{5}{ }^{5}$ cited in Table 3, provide supporting evidence for the use of gentamicin-citrate and sodium citrate-methylene blue-propylparaben, respectively. I agree with the statement by Filiopoulos et $\mathrm{al}^{1}$ that the ideal antimicrobial lock solution has not been identified to date, because several effective regimens have not been compared in head to head trials.

The authors go on to state that recent reviews appeared to advocate the use of lock solutions containing low concentrations of citrate in conjunction with taurolidine as the best choice at the moment and cite articles by Betjes ${ }^{6}$ and by Lok et al. ${ }^{7}$ It is true that Betjes, ${ }^{6}$ whose article was published online before the studies by Moran et $\mathrm{al}^{4}$ and Maki et al, ${ }^{5}$ stated that such combinations are theoretically the best choice at the moment, despite the fact that they have not been widely used and questions remain regarding their efficacy. However, the article by Lok et $\mathrm{al}^{7}$ does not make such a claim and points out that several recent trials using such combinations found no difference in the time to first CLABSI and that increasing use of thrombolytic therapy has been reported with the combination of citrate-taurolidine. Lok et $\mathrm{al}^{7}$ did mention that other novel antimicrobial lock solutions are promising and cited articles dealing with sodium citrate-methylene bluepropylparaben, ethanol, and ethanol-citrate. Finally, I agree with Filiopoulos et $\mathrm{al}^{1}$ that additional comparative studies are needed to identify the ideal lock solution.

\section{ACKNOWLEDGMENTS}

Potential conflicts of interest. J.M.B. has served as a consultant to $3 \mathrm{M}$ and has received research support and honoraria for activities unrelated to central line-associated infections. All authors submitted the ICMJE Form for Disclosure of Potential Conflicts of Interest, and the conflicts that the editors consider relevant to this article are disclosed here.

John M. Boyce, $\mathrm{MD}^{1,2}$

Affiliations: 1. Director, Hospital Epidemiology and Infection Control, Yale-New Haven Hospital, New Haven, Connecticut; 2. Clinical Professor of Medicine, Yale University School of Medicine, New Haven, Connecticut.

Address correspondence to John M. Boyce, MD, Hospital Epidemiology and Infection Control, Yale-New Haven Hospital, 20 York Street, New Haven, CT (john.boyce@ynhh.org).

Infect Control Hosp Epidemiol 2013;34(3):330

(C) 2013 by The Society for Healthcare Epidemiology of America. All rights reserved. 0899-823X/2013/3403-0020\$15.00. DOI: $10.1086 / 669568$

\section{REFERENCES}

1. Filiopoulos V, Hadjiyannakos D, Vlassopoulos D. Antimicrobial lock solutions in the prevention of catheter-related bloodstream infections in patients receiving hemodialysis. Infect Control Hosp Epidemiol 2012;34:329-330 (in this issue).

2. Boyce JM. Prevention of central line-associated bloodstream infection in hemodialysis patients. Infect Control Hosp Epidemiol 2012;33:936-944.

3. Filiopoulos V, Hadjiyannakos D, Koutis I, et al. Approaches to prolong the use of uncuffed hemodialysis catheters: results of a randomized trial Am J Nephrol 2011;33:260-268.

4. Moran J, Sun S, Khababa I, Pedan A, Doss S, Schiller B. A randomized trial comparing gentamicin/citrate and heparin locks for central venous catheters in maintenance hemodialysis patients Am J Kidney Dis 2012;59(1):102-107.

5. Maki DG, Ash SR, Winger RK, Lavin P; AZEPTIC Trial Investigators. A novel antimicrobial and antithrombotic lock solution for hemodialysis catheters: a multi-center, controlled, randomized trial. Crit Care Med 2011;39:613-620.

6. Betjes MGH. Prevention and management of catheter-related infection in patients on hemodialysis Nat Rev Nephrol 2011;7:257265.

7. Lok CE, Mokrzycki MH. Prevention and management of catheter-related infection in hemodialysis patients Kidney Int 2011; 79:587-598. 